\title{
WAVE PACKET PSEUDOMODES OF VARIABLE COEFFICIENT DIFFERENTIAL OPERATORS
}

\author{
LLOYD N. TREFETHEN
}

\begin{abstract}
The pseudospectra of nonselfadjoint linear ordinary differential operators with variable coefficients are considered. It is shown that when a certain winding number or twist condition is satisfied, closely related to Hörmander's commutator condition for partial differential equations, $\varepsilon$-pseudoeigenfunctions of such operators for exponentially small values of $\varepsilon$ exist in the form of localized wave packets. In contrast to related results of Davies and of Dencker, Sjöstrand, and Zworski, the symbol need not be smooth.
\end{abstract}

\section{Introduction}

Certain differential and pseudodifferential operators with variable coefficients have exponentially large resolvent norms, with respect to a parameter, in regions of the complex plane far from the spectrum. These large norms are explained by the existence of pseudoeigenfunctions in the form of localized wave packets which, although they may not satisfy the eigenvalue equation or the boundary conditions exactly, satisfy them approximately with an exponentially small error. Another way to describe this phenomenon is to say that these operators have $\varepsilon$-pseudospectra that extend over regions of the complex plane far from the spectrum, even for exponentially small values of $\varepsilon$. Applications in which such effects may be important include 'ghost solutions' of ordinary differential equations [22], non-Hermitian quantum mechanics $[\mathbf{3}, \mathbf{4}, \mathbf{5}, \mathbf{1 5}, \mathbf{1 6}]$, the theory of dichotomy for ordinary differential equations and their numerical approximation $[\mathbf{2}, \mathbf{9}, \mathbf{1 2}, \mathbf{1 4}, \mathbf{3 0}]$, fluid dynamics $[\mathbf{5}$, $\mathbf{8}, \mathbf{1 3}, \mathbf{3 4}, \mathbf{4 4}]$ and the Lewy/Hörmander phenomenon of nonexistence of solutions to certain linear partial differential and pseudodifferential operators $[\mathbf{2 6}, \mathbf{2 8}, \mathbf{4 8}]$.

The fact that variable coefficient nonselfadjoint differential operators may have extended pseudospectra with wave packet pseudomodes was pointed out by Davies in $1999[\mathbf{1 5}, \mathbf{1 6}]$. Shortly thereafter, Zworski observed that Davies's discoveries could be related to long-established solvability results in the theory of PDE by Hörmander and others [26, 47]. Later publications stimulated by Davies's work include $[\mathbf{1}, \mathbf{7}, \mathbf{1 9}, \mathbf{2 1}, \mathbf{3 2}, \mathbf{4 8}]$. The most convenient framework for all of these results is a semiclassical formulation, in which differentiated terms contain a small parameter $h$ or its powers $[\mathbf{2 3}, \mathbf{2 9}]$. Depending on the problem and the method of proof, the meaning of "exponentially small" for such an operator may involve terms of order $O\left(M^{-1 / h}\right)$ for some $M>1$ or of order $O\left(h^{N}\right)$ for all $N>0$ as $h \rightarrow 0$.

The standard method of deriving results about wave packet pseudomodes is WKBJ or microlocal analysis, in which a wave packet is constructed that is localized with respect to both the space variables and their dual, the wave number 
vector. This approach applies to both differential and pseudodifferential operators in any number of space dimensions, provided that the coefficients are smooth; the typical assumption is $C^{\infty}$. The most important presentation of this approach to date is the recent artricle of Dencker, Sjöstrand and Zworski [21].

For the special case of ordinary differential operators, however, an alternative method of proof is available, based on consideration of intersections of certain subspaces, that requires no assumption of smooth dependence on the space variable. The purpose of this article is to apply this method to establish a fundamental theorem about the existence of exponentially good pseudomodes for such problems. Instead of smoothness, this theorem depends upon a condition involving winding numbers of the symbol. One might think that this replacement of one condition by another would be be just a technical matter, of little significance for applications, since the conclusions reached about exponentially good pseudomodes are in the end the same. However, we shall show that the difference is a substantial one ( $\$ 7)$. For problems with smooth coefficients that violate the winding number condition, the pseudospectral effects are structurally unstable and vanish when the coefficients are perturbed in a nonsmooth manner. By contrast, the effects associated with operators that satisfy the winding number condition are robust.

This article also has another purpose, and that is to relate these phenomena to analogous effects that have been investigated in the highly developed field of Toeplitz matrices and operators and their generalizations with variable or "twisted" coefficients. The consideration of winding numbers is standard in the Toeplitz literature, as is the focus of attention on the case of one space dimension (although there are generalizations to block Toeplitz matrices and operators and to BerezinToeplitz operators on manifolds [6]). The analogy can be portrayed at a high level by considering four fundamental classes of nonnormal operators:

\begin{tabular}{rcc} 
& $\begin{array}{c}\text { boundary } \\
\text { pseudomodes }\end{array}$ & $\begin{array}{c}\text { wave packet } \\
\text { pseudomodes }\end{array}$ \\
\cline { 2 - 3 } differential & Reddy 93 & Davies 99 \\
operators & Davies 00 & Dencker-Sjöstrand-Zworski 04 \\
\cline { 2 - 3 } Toeplitz and twisted & Landau 75 & $\begin{array}{c}\text { Trefethen-Chapman 04 } \\
\text { Borthwick-Uribe 04 }\end{array}$ \\
Toeplitz matrices & Reichel-Trefethen 92 & Borthwillo
\end{tabular}

In all four cases the resolvent norm grows exponentially for certain problems as an appropriate parameter is increased or decreased, throughout a region of the complex plane determined by the symbol curves of the operator and their winding numbers. For each, we have listed the authors and date of the two most important published references; citation details can be found in the Bibliography. The present contribution belongs to the upper-right cell of this $2 \times 2$ array. Mathematically it is an analogue for differential operators of the earlier work for twisted Toeplitz matrices by Trefethen and Chapman [43]. Both our formulation of the main theorem and its proof stay as close as possible to those in [43], even to the point of repeating wordings where possible. However, the reader who compares the two papers will find that the two developments differ in many details. 


\section{An elementary example}

We can see the essence of the matter in a simple example. Consider the operator

$$
A_{h}: u \mapsto h u_{x}+x u,
$$

where $h$ is a small parameter, acting on a dense subspace of $L^{2}[-1,1]$ of differentiable functions satisfying the boundary conditions $u(-1)=u(1)=0$. The equation $A_{h} u=\lambda u$ cannot be solved for any $\lambda \in \mathbf{C}$; the spectrum of $A_{h}$ is empty. However, the function

$$
u(x)=e^{-x^{2} / 2 h}
$$

satisfies $A_{h} u=0$, and it satisfies the boundary conditions up to an error $e^{-1 / 2 h}$. Alternatively we could note that $e^{-x^{2} / 2 h}-e^{-1 / 2 h}$ satisfies the boundary conditions and satisfies $A_{h} u=0$ up to an error no greater than $e^{-1 / 2 h}$

Thus we can say that the Gaussian (2.2) of width $O\left(h^{1 / 2}\right.$ ) (or more precisely the same function shifted by a constant) is an $\varepsilon$-pseudoeigenfunction corresponding to the $\varepsilon$-pseudoeigenvalue 0 for a value of $\varepsilon$ of size $O\left(M^{-1 / h}\right)$ for some $M>1$ as $h \rightarrow 0 .^{\dagger}$ Moreover, the same is true for any number $\lambda$ with $-1<\operatorname{Re} \lambda<1$, as is shown by the pseudoeigenfunction

$$
u(x)=e^{-(x-\lambda)^{2} / 2 h}=C e^{-(x-\operatorname{Re} \lambda)^{2} / 2 h} e^{i x \operatorname{Im} \lambda / h} .
$$

The situation is summarized in Fig. 1 for $h=0.02$. (This and the other figures in this article were computed numerically by spectral discretization [42] and the EigTool pseudospectra plotting system [46]; we shall not give details.) We see that the pseudospectra of $A_{h}$ approximate the strip $-1<\operatorname{Re} \lambda<1$, and for the particular value $\lambda=\frac{1}{2}+i$, the optimal pseudoeigenfunction comes very close to the predicted form: a wave packet centred at $x=\frac{1}{2}$ with wave number $1 / h=50$, i.e., wavelength $2 \pi / 50 \approx 0.13$.

Once we have proved our main theorem and expressed it in terms of winding numbers, this example will be interpretable as follows. We associate the operator $A_{h}$ of (2.1) with the $x$-dependent symbol

$$
f(x, k)=-i k+x, \quad x \in(-1,1),
$$

which maps the real $k$-axis onto the negatively-oriented vertical line $\operatorname{Re} f=x$ in the complex plane. We define the winding number of this symbol curve about any point $\lambda \in \mathbf{C}$ by completing it by a large semicircle traversed counterclockwise in the right half-plane. Thus for each $x$, the winding number is 0 if $\operatorname{Re} \lambda<x, 1$ if $\operatorname{Re} \lambda>x$, and undefined if $\operatorname{Re} \lambda=x$. As $x$ increases from -1 to 1 , each $\lambda$ with $-1<\operatorname{Re} \lambda<1$ experiences a decrease in winding number when $x$ passes through the value $\operatorname{Re} \lambda$. Theorem 1 ensures that each such value of $\lambda$ is an $\varepsilon$-pseudoeigenvalue of $A_{h}$ for a value of $\varepsilon$ of size $O\left(M^{-1 / h}\right)$ for some $M>1$ as $h \rightarrow 0$.

\footnotetext{
${ }^{\dagger}$ An $\varepsilon$-pseudoeigenvalue and corresponding $\varepsilon$-pseudoeigenfunction of an operator $A$ are a scalar $\lambda$ and a nonzero function $u$ satisfying $\|(A-\lambda) u\| \leq \varepsilon\|u\|$, and the $\varepsilon$-pseudospectrum of $A$ is the union of its spectrum and the set of all of its $\varepsilon$-pseudoeigenvalues, i.e., the set of all $\lambda \in \mathbf{C}$ with resolvent norm satisfying $\left\|(\lambda-A)^{-1}\right\| \geq \varepsilon^{-1}$, if we adopt the convention of writing $\left\|(\lambda-A)^{-1}\right\|=\infty$ if $\lambda$ is in the spectrum $[\mathbf{2 4}, \mathbf{4 0}]$.
} 

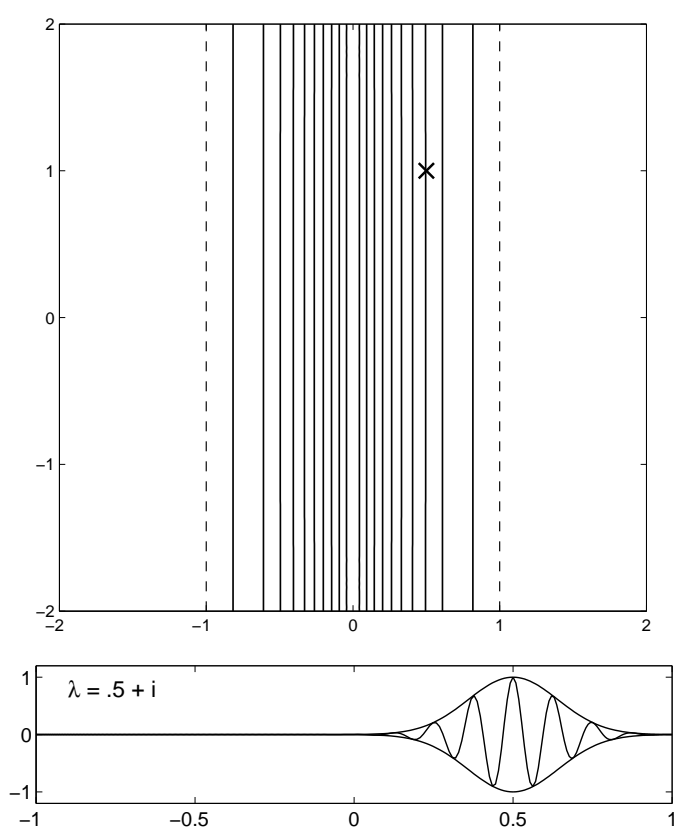

FIGURE 1. Above, $\varepsilon$-pseudospectra of the operator (2.1) with $h=0.02$ (the spectrum is

empty). From outside in, the contours correspond to $\varepsilon=10^{-1}, \ldots, 10^{-9}$. The figure confirms that the the resolvent norm $\left\|\left(\lambda-A_{h}\right)^{-1}\right\|$ grows exponentially as $h \rightarrow 0$ for any

$\lambda$ in the strip $-1 \leq \operatorname{Re} \lambda \leq 1$, marked by the dashed lines. Below, an optimal pseudoeigenfunction for $\lambda=\frac{1}{2}+i$ (marked by a cross in the top image) with $x_{*}=\frac{1}{2}$ and $k_{*}=-1$. (Both real part and envelope are shown.)

\section{Main theorem}

Let an interval $[a, b]$ be given, $a<b$, and for a small parameter $h>0$, let $D_{h}$ be the scaled derivative operator

$$
D_{h}=i h \frac{d}{d x}
$$

For some integer $n \geq 0$, let continuous coefficient functions

$$
c_{j}(x), \quad 0 \leq j \leq n
$$

be defined on $(a, b)$, which may or may not be smooth. We consider the family of linear operators $\left\{A_{h}\right\}, h>0$, defined by

$$
\left(A_{h} u\right)(x)=\sum_{j=0}^{n} c_{j}(x)\left(D_{h}^{j} u\right)(x), \quad a<x<b,
$$

together with arbitrary homogeneous boundary conditions at $x=a$ and $x=b$ (the details of the boundary conditions will not matter), acting in a suitable dense domain in $L^{p}[a, b]$ for some $p$ with $1 \leq p<\infty$.

Given $k \in \mathbf{C}$ and $h$, consider the function $v(x)=e^{-i k x / h}$ in $L^{p}[a, b]$. We can 
write $A_{h} v$ explicitly as

$$
\left(A_{h} v\right)(x)=\sum_{j=0}^{n} c_{j}(x) k^{j} v(x)
$$

In other words, we have

$$
\left(A_{h} v\right)(x)=f(x, k) v(x)
$$

where $f$ is the symbol of $\left\{A_{h}\right\}$, defined for $x \in(a, b)$ and $k \in \mathbf{C}$ by

$$
f(x, k)=\sum_{j=0}^{n} c_{j}(x) k^{j} .
$$

Our aim is to build wave packets localized near a particular $x_{*} \in(a, b)$. Let $x_{*}$ and $k_{*}$ be given, and define $\lambda=f\left(x_{*}, k_{*}\right)$. If $f$ were independent of $x$, the function

$$
v(x)=e^{-i k_{*} x / h}
$$

would satisfy the eigenfunction equation for $A_{h}$ with eigenvalue $\lambda$,

$$
A_{h} v=\lambda v
$$

though in general it would not be an eigenfunction because of the boundary conditions. If $f$ varies with $x$, however, we shall look for solutions to (3.3) near $x_{*}$ with the form of wave packets (exact solutions, not approximate), and if these decay exponentially away from $x_{*}$, they can be extended smoothly to zero so as to make exponentially good pseudoeigenfunctions, regardless of the boundary conditions.

Definition 1. The symbol $f=f(x, k)$ satisfies the twist condition at $x=$ $x_{*} \in(a, b), k=k_{*} \in \mathbf{R}$ if at this point it is differentiable with respect to $x$ with $\partial f / \partial k \neq 0$ and

$$
\operatorname{Im}\left(\frac{\partial f}{\partial x} / \frac{\partial f}{\partial k}\right)>0 .
$$

It satisfies the antitwist condition if it has the same properties with (3.4) replaced by

$$
\operatorname{Im}\left(\frac{\partial f}{\partial x} / \frac{\partial f}{\partial k}\right)<0
$$

Here is our main theorem.

THEOREM 1. Let $\left\{A_{h}\right\}$ be a family of variable coefficient differential operators on $[a, b]$ with symbol $f(x, k)$ as described above. Given $x_{*} \in(a, b)$ and $k_{*} \in \mathbf{R}$, define $\lambda=f\left(x_{*}, k_{*}\right)$, and suppose that the twist condition (3.4) or the antitwist condition (3.5) is satisfied. Suppose moreover that $f\left(x_{*}, k\right) \neq \lambda$ for all real $k \neq k_{*}$ and that $c_{n}\left(x_{*}\right) \neq 0$. Then there exist constants $C_{1}, C_{2}>0$ and $M>1$ such that for all sufficiently small $h$, there exists a nonzero pseudoeigenfunction $v^{(h)}$ that is exponentially good,

$$
\frac{\left\|\left(A_{h}-\lambda\right) v^{(h)}\right\|}{\left\|v^{(h)}\right\|} \leq M^{-1 / h} .
$$

If it is the twist condition that is satisfied, then $v^{(h)}$ can also be taken to be local- 


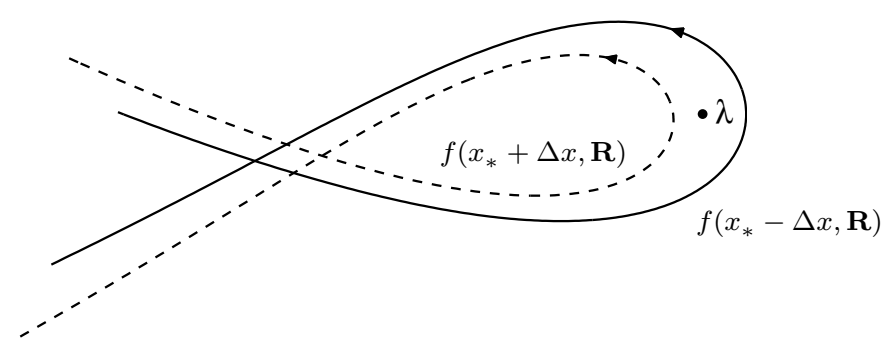

FiguRE 2. Winding number interpretation of Theorem 1 . If the curve $f(x, \mathbf{R})$ crosses $\lambda$ as $x$ increases through $x_{*}$ in such a way that the winding number about $\lambda$ is decreased, then there is an exponentially good wave packet pseudomode localized at $x_{*}$ with pseudoeigenvalue $\lambda$.

ized,

$$
\frac{\left|v^{(h)}(x)\right|}{\max _{x}\left|v^{(h)}(x)\right|} \leq C_{1} \exp \left(-C_{2}\left(x-x_{*}\right)^{2} / h\right)
$$

In what follows we shall discuss only the twist condition and localized pseudoeigenfunctions; the claim concerning the antitwist condition follows by considering the transposed operators, i.e., the complex conjugates of the adjoints. In essence this is a matter of reversing the dependence on $x$.

\section{Winding number interpretation}

The condition $f\left(x_{*}, k\right) \neq \lambda$ for $k \neq k_{*}$ of Theorem 1 might easily be overlooked, but it is important: it is the price we pay for not requiring $f$ to depend smoothly on $x$. This condition has a geometric interpretation. Given a symbol $f=f(x, k)$ and a particular value $x=x_{*}$, define the symbol curve of $f$ at $x_{*}$ to be the curve $f\left(x_{*}, \mathbf{R}\right)$, a subset of the complex plane. Given a number $\lambda \in \mathbf{C} \backslash f\left(x_{*}, \mathbf{R}\right)$, we wish to define the winding number of $f$ with respect to $\lambda$. Since $f\left(x_{*}, \mathbf{R}\right)$ is not a closed curve, this requires some care. A suitable approach is to make use of contours $\Gamma_{R}$ consisting of the real interval $[-R, R]$ closed by a semicircle of radius $R$ in the upper half complex plane. We consider the winding number of $f$ about $\lambda$ associated with this closed contour traversed in the usual anticlockwise direction, and we define the winding number $I\left(f, x_{*}, \lambda\right)$ to be the limiting winding number of $f\left(x_{*}, \Gamma_{R}\right)$ about $\lambda$ obtained for all sufficiently large $R$. If $\lambda \in f\left(x_{*}, \mathbf{R}\right), I\left(f, x_{*}, \lambda\right)$ is undefined.

It is readily verified that the conditions of Theorem 1 have the following geometric interpretations:

- The symbol curve at $x_{*}$ passes just once through $\lambda$;

- As $x$ increases through $x_{*}$, the winding number about $\lambda$ decreases (twist condition) or increases (antitwist condition) by 1.

One can use these conditions to assess quickly where the pseudospectra of a variable coefficient differential operator will lie (Fig. 2). One imagines the symbol curve moving about the complex plane as $x$ increases from $a$ to $b$. The pseudospectra fill the region swept out by this moving curve.

In fact, we can make an explicit connection between the winding number $I=$ $I(f, \lambda, x)$ at a point $x$ where it is defined and the eigenvalues of the associated 
transfer matrix $C(x)$ introduced below in (5.1)-(5.2). By the principle of the argument of complex analysis, the symbol polynomial $f$ at $x$ has exactly $I$ zeros in the upper half-plane. In Lemmas 1 and 2, this will correspond to a space of amplified functions of dimension $I$ and a space of attenuated functions of dimension $n-I$.

\section{Proof of Theorem 1}

In this section we prove Theorem 1 . The essential idea, involving a nonempty intersection of subspaces of functions decaying to the left and the right that satisfy the eigenvalue equation exactly in an interval, is summarized at the end.

Given an $n$-times differentiable function $v(x)$, define

$$
v^{(j)}=D_{h}^{j} v, \quad 0 \leq j \leq n
$$

and

$$
\mathbf{v}=\left(v, v^{(1)}, \ldots, v^{(n-1)}\right)^{T} .
$$

Then (3.3) can be written

or more compactly,

$$
D_{h} \mathbf{v}=\left(\begin{array}{ccccc}
0 & 1 & & & \\
& 0 & 1 & & \\
& & 0 & \ddots & \\
& & & \ddots & 1 \\
e_{0}(x) & e_{1}(x) & e_{2}(x) & \ldots & e_{n-1}(x)
\end{array}\right) \mathbf{v}
$$

$$
D_{h} \mathbf{v}(x)=C(x) \mathbf{v}(x),
$$

where $e_{j}(x)=-c_{j}(x) / c_{n}(x)$ for $1 \leq j \leq n$ and $e_{0}(x)=\left(\lambda-c_{0}(x)\right) / c_{n}(x)$. Thus we have a first-order vector-valued linear ODE with continuous coefficients defined throughout the neighborhood of $x_{*}$ where $c_{n}(x) \neq 0$, with $n$ linearly independent solutions throughout this region (see e.g. Section 3.6 of [11]). We may associate equation (5.1) frozen at the point $x_{*}$ with the characteristic polynomial

$$
p(k)=\sum_{j=0}^{n} e_{j}\left(x_{*}\right) k^{j} .
$$

For $\kappa \in \mathbf{C}$, the frozen-coefficient ODE has a solution $\mathbf{v}(x)=\left(v, v^{(1)}, \ldots, v^{(n-1)}\right)^{T}$ with

$$
v=v(x)=e^{-i \kappa x / h}
$$

if and only if $\kappa$ satisfies $p(\kappa)=0$. The polynomial $p$ has exactly $n$ roots $\left\{\kappa_{\ell}\right\}$ counted with multiplicity for $x$ sufficiently close to $x_{*}$. Each root with $\operatorname{Im} \kappa>0$ corresponds to exponential growth as $x$ increases and exponential decay as $x$ decreases, and for $\operatorname{Im} \kappa<0$ the pattern is reversed. As mentioned at the end of the last section, if the winding number $I=I\left(f, \lambda, x_{*}\right)$ of $f$ about $\lambda$ at $x_{*}$ is well-defined, then there will be $I$ roots of the former type and $n-I$ roots of the latter type.

Of course, the problem at hand involves variable coefficients. Our choice of $\lambda$ will ensure that one root has $\operatorname{Im} \kappa=0$ and passes from above to below the real axis as $x$ increases through $x_{*}$, and thus corresponds to exponential decay in both directions. We begin in Lemma 1, however, with a result based on the assumption that the 
roots are separated from the axis. We work with a fixed range of values of $x$ around $x_{*}$, chosen narrow enough so that the coefficients $\left\{c_{\ell}(x)\right\}$ vary sufficiently little and so that $c_{n}(x) \neq 0$. We consider the variable coefficient ODE

$$
D_{h} \mathbf{v}=(C+E(x)) \mathbf{v},
$$

where $C=C\left(x_{*}\right)$, and let $S_{h}(x)$ denote its solution matrix or matrizant: if $\mathbf{v}\left(x_{*}\right)$ is an $n$-vector, then $\mathbf{v}(x)=S_{h}\left(x-x_{*}\right) \mathbf{v}\left(x_{*}\right)$ is the corresponding unique solution of (5.5) near $x_{*}$. (If $E(x) \equiv 0, S_{h}(x)=\exp (-i C x / h)$.) We first show that under suitable hypotheses, the solutions to this equation attenuate and amplify certain vectors exponentially. This is a basic result with the flavour of the stable manifold theorem in dynamical systems. Afterwards we shall refine it to the more specialized Lemma 2, analogous to the centre manifold theorem, which is actually the one needed to prove Theorem $1[\mathbf{3 8}, \mathbf{4 5}]$.

Lemma 1. Let $C$ be an $(\eta+\nu) \times(\eta+\nu)$ matrix $(\eta, \nu \geq 0, \eta+\nu \geq 1)$ that has $\eta$ eigenvalues $\kappa$ with $\operatorname{Im} \kappa<\rho<0$ and $\nu$ eigenvalues with $\operatorname{Im} \kappa>R>0$, let $E(x)$ be a continuous matrix function with $\|E(x)\| \leq \varepsilon$, let $S_{h}(x)$ be the solution matrix for (5.5) for $x$ near $x_{*}=0$, and let $\Delta x>0$ be fixed. There exists $\varepsilon>0$, independent of $h$, such that the solution matrices $\left\{S_{h}(\Delta x)\right\}$ separate $\mathbf{C}^{\eta+\nu}$ into exponentially amplified and attenuated subspaces in the following sense: for each sufficiently small $h>0$, there is an $\eta$-dimensional subspace $\mathcal{S}_{\eta} \subseteq \mathbf{C}^{\eta+\nu}$ such that

$$
\left\|S_{h}(\Delta x) \mathbf{u}\right\| \leq e^{\rho \Delta x / h}\|\mathbf{u}\| \quad \forall \mathbf{u} \in \mathcal{S}_{\eta}
$$

and a $\nu$-dimensional subspace $\mathcal{S}_{\nu} \subseteq \mathbf{C}^{\eta+\nu}$ such that

$$
\left\|S_{h}(\Delta x) \mathbf{u}\right\| \geq e^{R \Delta x / h}\|\mathbf{u}\| \quad \forall \mathbf{u} \in \mathcal{S}_{\nu} .
$$

Proof. We shall see that $\mathcal{S}_{\nu}$ can be chosen independently of $h$, since almost all vectors lead to exponential growth, but $\mathcal{S}_{\eta}$ must depend on $h$, since exponential decay is more delicate.

The first step is to reduce $C$ to block-diagonal form. There exists a nonsingular matrix $X$ such that

$$
X C X^{-1}=\left(\begin{array}{cc}
G_{\nu} & 0 \\
0 & G_{\eta}
\end{array}\right),
$$

where $G_{\nu}$ has dimension $\nu$ and amplifies all $\nu$-vectors,

$$
\left\|e^{-i s G_{\nu}} \mathbf{v}\right\| \geq e^{s T}\|\mathbf{v}\| \quad \forall \mathbf{v} \in \mathbf{C}^{\nu}, \forall s>0
$$

and $G_{\eta}$ has dimension $\eta$ and shrinks all $\eta$-vectors,

$$
\left\|e^{-i s G_{\eta}} \mathbf{w}\right\| \leq e^{s \tau}\|\mathbf{w}\| \quad \forall \mathbf{w} \in \mathbf{C}^{\eta}, \forall s>0 ;
$$

we may choose any $T>R$ which is smaller than the imaginary parts of all the eigenvalues of $C$ in the upper half-plane, and any $\tau<\rho$ which is larger than the imaginary parts of all the eigenvalues of $C$ in the lower half-plane. If (5.5) is leftmultiplied by $X$, we obtain a new differential equation for the variable $X \mathbf{v}$ involving the matrix (5.8); the norms of $X$ and $X^{-1}$ introduce fixed constants and thus have no effect on the small- $h$ assertion. Since $E(x)$ becomes $X E(x) X^{-1}$ in this process, the norm bound $\varepsilon$ has to be adjusted by the condition number $\kappa(X)=\|X\|\left\|X^{-1}\right\|$. All this is straightforward, and rather than encumber the rest of the argument with $X$ and associated details, let us assume from now on, without loss of generality, 
that $C$ itself has block diagonal form to begin with,

$$
C=\left(\begin{array}{cc}
G_{\nu} & 0 \\
0 & G_{\eta}
\end{array}\right)
$$

with $G_{\nu}$ and $G_{\eta}$ satisfying (5.9) and (5.10).

We identify first a growing space $S_{\nu}$ as in (5.7). Define $\delta, \varepsilon>0$ by

$$
\delta=\min \left\{\frac{T-R}{-\tau}, 1\right\}, \quad \varepsilon=\frac{-\tau \delta}{4},
$$

and let $\mathcal{G} \subseteq \mathbf{C}^{\eta+\nu}$ denote the cone of all vectors $\mathbf{u}=\left(\mathbf{v}^{T}, \mathbf{w}^{T}\right)^{T} \in \mathbf{C}^{\eta+\nu}$ satisfying

$$
\|\mathbf{w}\| \leq \delta\|\mathbf{v}\|
$$

Consider what happens when $S_{h}(x)$ acts on a vector $\mathbf{u}=\left(\mathbf{v}^{T}, \mathbf{w}^{T}\right)^{T} \in \mathcal{G}$ :

$$
\left(\begin{array}{c}
\tilde{\mathbf{v}} \\
\tilde{\mathbf{w}}
\end{array}\right)=\left(\begin{array}{c}
\tilde{\mathbf{v}}(x) \\
\tilde{\mathbf{w}}(x)
\end{array}\right)=S_{h}(x)\left(\begin{array}{c}
\mathbf{v} \\
\mathbf{w}
\end{array}\right)
$$

assuming (without loss of generality) $\|\mathbf{v}\|=1$. At $x=0$ we have, by (5.5) and (5.11),

$$
\frac{d}{d(x / h)}\left(\begin{array}{c}
\tilde{\mathbf{v}} \\
\tilde{\mathbf{w}}
\end{array}\right)=\frac{d}{d(x / h)} S_{h}(x)\left(\begin{array}{c}
\mathbf{v} \\
\mathbf{w}
\end{array}\right)=\left[\left(\begin{array}{cc}
-i G_{\nu} & 0 \\
0 & -i G_{\eta}
\end{array}\right)-i E(0)\right]\left(\begin{array}{c}
\mathbf{v} \\
\mathbf{w}
\end{array}\right),
$$

from which we calculate, using (5.9),

$$
\frac{d\|\tilde{\mathbf{v}}\|}{d(x / h)} \geq T-\varepsilon-\varepsilon \delta \geq T-2 \varepsilon=T+\frac{\tau \delta}{2} \geq T-\frac{(T-R)}{2}>R,
$$

and, using (5.10),

$$
\frac{d\|\mathbf{w}\|}{d(x / h)} \leq \tau\|\mathbf{w}\|+\varepsilon+\varepsilon\|\mathbf{w}\| \leq \tau\|\mathbf{w}\|+2 \varepsilon=\tau\left(\|\mathbf{w}\|-\frac{\delta}{2}\right) .
$$

Thus locally $S_{h}(x)$ increases the $\mathbf{v}$ component of a vector at a rate greater than $R$ and decreases the $\mathbf{w}$ component, if the latter has norm greater than $\delta / 2$. These observations imply that $S_{h}(x)$ maps $\mathcal{G}$ into itself and that (5.7) holds for sufficiently small $h$ for all $\mathbf{u} \in \mathcal{G}$. Finally, we note that $\mathcal{G}$ contains the $\nu$-dimensional invariant subspace associated with $G_{\nu}$, i.e., the subspace spanned by the $\nu$ vectors $\mathbf{e}_{1}, \mathbf{e}_{2}, \ldots, \mathbf{e}_{\nu} \in \mathbf{C}^{\eta+\nu}$. Thus this subspace is a suitable choice for $S_{\nu}$.

To find a decaying space $S_{\eta}$ for (5.6), we consider the inverse solution operator $S_{h}(\Delta x)^{-1}$, with

$$
\left(\begin{array}{c}
\mathbf{v} \\
\mathbf{w}
\end{array}\right)=S_{h}(\Delta x)^{-1}\left(\begin{array}{c}
\tilde{\mathbf{v}}(\Delta x) \\
\tilde{\mathbf{w}}(\Delta x)
\end{array}\right) .
$$

By the same argument as before, $S_{h}(\Delta x)^{-1}$ has a growing space $\mathcal{T}$ of dimension $\eta$, and we take $\mathcal{S}_{\eta}=S_{h}(\Delta x)^{-1} \mathcal{T}$.

The next lemma is a variant of Lemma 1 designed for systems that have a root passing through real axis, as occurs at the center of a wave packet pseudomode.

LEMma 2. Let $\{C(x)\}$ be a family of $(\eta+\nu+1) \times(\eta+\nu+1)$ matrices $(\eta, \nu \geq 0)$ depending differentiably on $x$ at $x=x_{*}$. Assume that $C_{*}=C\left(x_{*}\right)$ has $\eta$ eigenvalues with $\operatorname{Im} \kappa<\rho<0, \nu$ eigenvalues with $\operatorname{Im} \kappa>R>0$, and one eigenvalue $\mu_{*}$ with $\operatorname{Im} \mu_{*}=0$. Assume further that $d \operatorname{Im} \mu / d x<0$ at $x=x_{*}$, where $\mu=\mu(x)$ is the 
eigenvalue of $C(x)$ that converges to $\mu_{*}$ as $x \rightarrow x_{*}$. For $x$ near $x_{*}$ and $h>0$, consider the solution matrix $S_{h}(x)$ as before. There exist $\Delta x>0$ and $M>0$, independent of $h$, such that these operators separate $\mathbf{C}^{\eta+\nu+1}$ into exponentially amplified and attenuated subspaces in the following sense: for each sufficiently small $h$, there is an $(\eta+1)$-dimensional subspace $\mathcal{S}_{\eta} \subseteq \mathbf{C}^{\eta+\nu+1}$ such that

$$
\left\|S_{h}(\Delta x) \mathbf{u}\right\| \leq e^{-M / h}\|\mathbf{u}\| \quad \forall \mathbf{u} \in \mathcal{S}_{\eta}
$$

and a $\nu$-dimensional subspace $\mathcal{S}_{\nu} \subseteq \mathbf{C}^{\eta+\nu+1}$ such that

$$
\left\|S_{h}(\Delta x) \mathbf{u}\right\| \geq e^{M / h}\|\mathbf{u}\| \quad \forall \mathbf{u} \in \mathcal{S}_{\nu} .
$$

Proof. It is only (5.12) we shall need, not (5.13), so this is the claim we shall prove. As in the final paragraph of the proof of Lemma 1, to show that $S_{h}(\Delta x)$ has a decaying subspace of dimension $\eta+1$, we shall show that $S_{h}(\Delta x)^{-1}$ has a growing subspace of this dimension. Our first step, as in the proof of Lemma 1, is to assume without loss of generality that $-C\left(x_{*}\right)$ has the block-diagonal form

$$
-C\left(x_{*}\right)=\left(\begin{array}{ccc}
G_{\nu} & 0 & 0 \\
0 & -\mu_{*} & 0 \\
0 & 0 & G_{\eta}
\end{array}\right),
$$

where $G_{\nu}$ has dimension $\nu$ and amplifies all $\nu$-vectors as in (5.9), $G_{\eta}$ has dimension $\eta$ and shrinks all $\eta$-vectors as in (5.10), and $\operatorname{Im} \mu_{*}=0$, with $\tau<0<T$.

Without loss of generality we assume $x_{*}=0$, as in Lemma 1 .

The proof rests on an observation of linear algebra: if a diagonal matrix is perturbed by $O(\varepsilon)$ in the off-diagonal positions, its eigenvalues of multiplicity 1 change by only $O\left(\varepsilon^{2}\right)$. (One can prove this by considering the characteristic polynomial.) We exploit this phenomenon as follows. Our task is to consider the solution operator associated with a set of matrices

$$
-C(x)=\left(\begin{array}{ccc}
G_{\nu} & 0 & 0 \\
0 & c(x) & 0 \\
0 & 0 & G_{\eta}
\end{array}\right)+E(x)
$$

with $\|E(x)\|=O(x)$, where $E(x)$ is constructed to have a zero entry in the middle position $(\nu+1, \nu+1)$ and $c(x)$ is defined accordingly. Since $\mu(x)=\mu_{*}+x(d \mu / d x)+$ $o(x)$, it follows from the fact of linear algebra just mentioned that $c(x)=-\mu_{*}-$ $x(d \mu / d x)+o(x)$. Choose $\gamma$ to be a constant in the range $0<\gamma<-d \operatorname{Im} \mu / d x$, and collect the upper-left $(\eta+1) \times(\eta+1)$ block of $C(x)$ into a single matrix $G_{\nu+1}(x)$. Then for some $\sigma>0$ and $x_{\max }>0$ we have

and

$$
-C(x)=\left(\begin{array}{cc}
G_{\nu+1}(x) & E_{1}(x) \\
E_{2}(x) & G_{\eta}(x)
\end{array}\right), \quad\left\|E_{1}\right\|,\left\|E_{2}\right\| \leq \sigma x
$$

$$
\begin{array}{cc}
\left\|e^{-i s G_{\nu+1}(x)} \mathbf{v}\right\| \geq e^{s \gamma x}\|\mathbf{v}\| & \forall \mathbf{v} \in \mathbf{C}^{\nu+1}, \forall s>0, \\
\left\|e^{-i s G_{\eta}(x)} \mathbf{w}\right\| \leq e^{s \tilde{\tau}}\|\mathbf{w}\| & \forall \mathbf{w} \in \mathbf{C}^{\eta}, \forall s>0
\end{array}
$$

for some $\tilde{\tau}<0$ and all $x \leq x_{\max }$. (The reasoning that establishes (5.15) is as follows: if $G$ is a matrix and $\alpha$ is the minimal imaginary part of the field of values of $G$, i.e., the minimal eigenvalue of $i\left(G-G^{*}\right) / 2$, then $\left\|e^{-i s G} \mathbf{v}\right\| \geq e^{\alpha s}\|\mathbf{v}\|$ for any $\mathbf{v}$; here, 
by the $O\left(\varepsilon^{2}\right)$ observation above, $\alpha$ increases faster than $\gamma x$ as $x$ increases from 0 .) We now follow estimates as in the proof of Lemma 1. Define

$$
\delta=\frac{\gamma}{2 \sigma}, \quad \Delta x=\min \left\{x_{\max }, \frac{-\delta \tilde{\tau}}{\sigma}\right\} .
$$

Let $\mathcal{G} \subseteq \mathbf{C}^{\eta+\nu+1}$ be the cone of all vectors $\mathbf{u}=\left(\mathbf{v}^{T}, \mathbf{w}^{T}\right)^{T} \in \mathbf{C}^{\eta+\nu+1}$ satisfying

$$
\|\mathbf{w}\| \leq \delta\|\mathbf{v}\| \text {. }
$$

Consider how a vector $\mathbf{u}=\left(\mathbf{v}^{T}, \mathbf{w}^{T}\right)^{T} \in \mathcal{G}$ evolves locally near a point $x \in[0, \Delta x]$, assuming without loss of generality $\|\mathbf{v}\|=1$. We have

$$
\frac{d}{d(x / h)}\left(\begin{array}{c}
\tilde{\mathbf{v}} \\
\tilde{\mathbf{w}}
\end{array}\right)=\left(\begin{array}{cc}
-i G_{\nu+1}(x) & -i E_{1}(x) \\
-i E_{2}(x) & -i G_{\eta}(x)
\end{array}\right)\left(\begin{array}{c}
\mathbf{v} \\
\mathbf{w}
\end{array}\right)
$$

from which we calculate

$$
\frac{d\|\tilde{\mathbf{v}}\|}{d(x / h)} \geq \gamma x-\sigma \delta x \geq \frac{\gamma x}{2}
$$

and

$$
\frac{d\|\tilde{\mathbf{w}}\|}{d(x / h)} \leq \sigma \Delta x+\tilde{\tau}\|\tilde{\mathbf{w}}\| \leq \tilde{\tau}(\|\tilde{\mathbf{w}}\|-\delta) .
$$

These estimates show that $S_{h}(x)^{-1}$ maps $\mathcal{G}$ into itself and increases the norm of the upper part of the vector at a rate at least $\gamma x / 2 h$. It follows that if $\mathbf{u} \in \mathcal{G}$, then $S_{h}(\Delta x)^{-1} \mathbf{u} \in \mathcal{G}$ and $\left\|S_{h}(\Delta x)^{-1} \mathbf{u}\right\| \geq e^{M / h}\|\mathbf{u}\|$ for all sufficiently small $h$, for some constant $M \approx \gamma \Delta x / 4$. Finally, $\mathcal{G}$ contains the $(\nu+1)$-dimensional invariant subspace associated with $G_{\nu+1}$, which is accordingly a suitable choice of $S_{\nu}$.

Proof of Theorem 1. To establish Theorem 1, we construct a wave packet in an interval $\left[x_{*}-\Delta x, x_{*}+\Delta x\right]$ that satisfies the eigenvalue equation (3.3) exactly in that interval and is also localized in the sense of (3.7) there. We do this by applying Lemma 2 in both directions, moving both right and left from $x=x_{*}$, taking advantage of the equivalence between (3.3) and (5.1).

First let us move from $x_{*}$ to the right. The hypothesis of Theorem 1 that $f\left(x_{*}, k\right) \neq \lambda$ for all real $k \neq k_{*}$ ensures that the matrix $C\left(x_{*}\right)$ has just a single eigenvalue $\mu_{*}$ on the real axis, as required in Lemma 2. The twist condition ensures that $d \operatorname{Im} \mu / d x<0$ at $x=x_{*}$, and the hypotheses $c_{n}\left(x_{*}\right) \neq 0$ ensures that $C(x)$ is well-defined for all $x$ sufficiently close to $x_{*}$. The matrices $S_{h}(x)$ of Lemma 2 are then the solution operators that transfer an $n$-vector of data $\left(v, v^{(1)}, \ldots, v^{(n-1)}\right)$ to the right a distance $x$. According to Lemma 2 , for some $\eta \geq 0$ there is an $(\eta+1)$ dimensional subspace $\mathcal{S}_{\eta}^{\text {(right) }} \subseteq \mathbf{C}^{m+n}$ of such data vectors that generate solutions in $\left[x_{*}, x_{*}+\Delta x\right]$ that are exponentially small in the sense of (5.12) at the end of that interval. By the same argument, though this is not spelled out in the statement of Lemma 2, these solutions have the Gaussian decay behavior described by (3.7).

Similarly, by an obvious symmetry, we can apply Lemma 2 moving from $x_{*}$ to the left. We conclude that with $\nu=m+n-1-\eta$ there is a $(\nu+1)$-dimensional subspace $\mathcal{S}_{\eta}^{(\text {left })} \subseteq \mathbf{C}^{m+n}$ of data vectors that generate solutions in $\left[x_{*}-\Delta x, x_{*}\right]$ with the appropriate Gaussian decay.

Now we take the intersection of these two subspaces. Their dimensions are $\eta+1$ and $\nu+1=m+n-\eta$, and they are subspaces of $\mathbf{C}^{m+n}$. It follows that $\mathcal{S}_{\eta}^{\text {(right) }} \cap \mathcal{S}_{\eta}^{\text {(left) }}$ is a subspace of dimension at least 1 (in fact, it will be exactly 1 ) of vectors that 
generate solutions of the eigenvalue equation in $\left[x_{*}-\Delta x, x_{*}+\Delta x\right]$ that decay appropriately on both sides of $x_{*}$.

Thus there exists a vector that exactly satisfies the eigenvalue equation in $\left[x_{*}-\right.$ $\left.\Delta x, x_{*}+\Delta x\right]$ and has the necessary decay there. Near $x_{*}-\Delta x$ and $x_{*}+\Delta x$, such a vector will be exponentially small. By extending it smoothly to 0 outside this interval, we obtain a pseudoeigenfunction $v^{(h)}$ satisfying both (3.6) and (3.7).

\section{Examples}

We shall present six numerical examples, the first three fitting the framework of Theorem 1 and the others having a double-crossing symbol curve, so that only the theorems of Dencker, et al. [21] can be applied. The next section will comment on the significance of this distinction.

Our first example is a variable coefficient advection-diffusion operator from a paper of Cossu and Chomaz [13]. The operator (after some simplification) is

$$
A_{h} u=h^{2} u_{x x}+h u_{x}+\left(\frac{1}{4}-x^{2}\right) u
$$

for $x \in \mathbf{R}$, and the eigenvalues and eigenfunctions are known explicitly:

$$
\lambda_{n}=-(2 n+1) h, \quad u_{n}=e^{-\left(x+x^{2}\right) / 2 h} H_{n}(x / \sqrt{h})
$$

for $n=1,2,3, \ldots$, where $H_{n}$ is the $n$th Hermite polynomial [10]. Pseudospectra for the case $h=0.02$ are shown in Fig. 3, and we see that the resolvent norm is exponentially large in the region of the complex plane bounded by the parabola $\operatorname{Re} \lambda=\frac{1}{4}-(\operatorname{Im} \lambda)^{2}$. To explain this hehavior we note that the symbol is

$$
f(x, k)=-k^{2}-i k+\left(\frac{1}{4}-x^{2}\right),
$$

which implies that the symbol curves are parabolas adjusted by the variable offset $\frac{1}{4}-x^{2}$. The winding number about a value $\lambda$ decreases from 1 to 0 as this curve crosses $\lambda$ from left to right, which occurs for a negative value of $x$, and this explains why the pseudoeigenfunction in the figure sits in the left half of the domain.

This example illustrates some behavior of widespread physical importance. The operator (6.1) can be interpreted as a standard advection-diffusion process coupled with a factor that introduces exponential amplification for $|x|<1 / 2$ and exponential attenuation for $|x|>1 / 2$. It is clear that the associated time-dependent process $u_{t}=A_{h} u$ must be susceptible to transient growth of order $O\left(C^{1 / h}\right)$ on a time scale $O\left(h^{-1}\right)$ for some $C>1$, for a pulse will grow exponentially during the time of order $O\left(h^{-1}\right)$ that it spends passing through the amplification region. Cossu and Chomaz relate this transient effect to the local convective instability of fluid flows in unbounded domains, including wakes, jets, and boundary layers. Alternatively, it is implied by the appearance of exponentially large resolvent norms in the right half-plane; see Theorem 5 of [40].

Our second example is a more exotic advection-diffusion operator investigated by Benilov, O'Brien and Sazonov [5]. These authors consider the instability of a thin viscous liquid film on the inner surface of a rotating cylinder in an approximation in which gravitational effects are included but inertial and capillary effects are ignored. They reduce their problem (again after some simplification) to the operator

$$
A_{h} u=h^{2} \sin (x) u_{x x}+h u_{x}
$$

with periodic boundary conditions on $[-\pi, \pi]$, with symbol $f(x, k)=-\sin (x) k^{2}-i k$. 

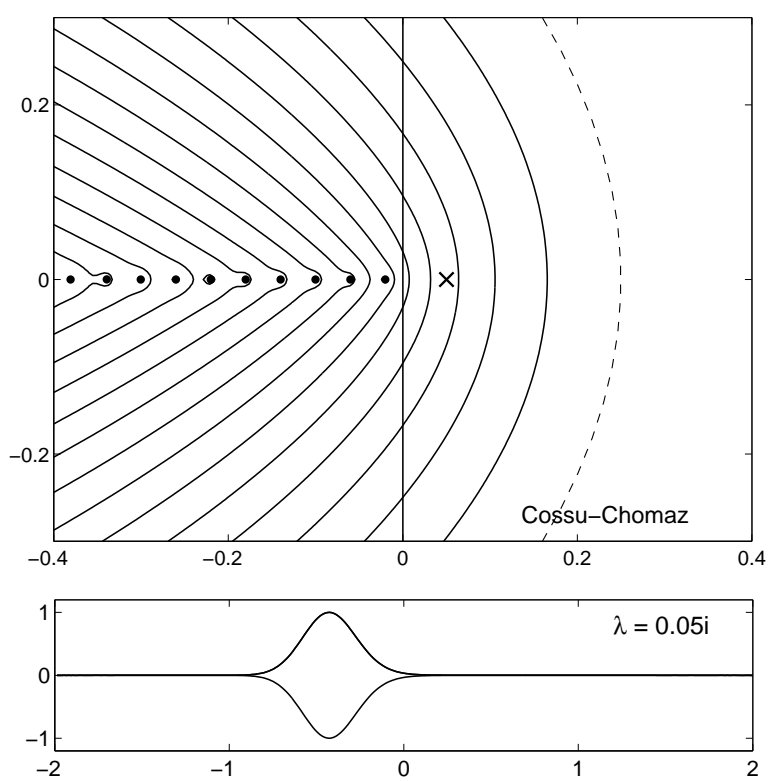

Figure 3. Above, eigenvalues and $\varepsilon$-pseudospectra of the operator (6.4) of Cossu and Chomaz $[\mathbf{1 3}]$ with $h=0.02, \varepsilon=10^{-2}, \ldots, 10^{-15}$. By Theorem 1 , the resolvent norm $\left\|(\lambda-A)^{-1}\right\|$ grows exponentially as $h \rightarrow 0$ for any $\lambda$ lying in the region to the left of the dashed parabola. Below, an optimal pseudoeigenfunction for $\lambda=0.05 i$ (marked by a cross in the top image) with central position and wave number $x_{*}=-5^{-1 / 2} \approx-0.45$ and

$$
k_{*}=0 \text {. }
$$

An unusual feature here is that for two values of $x$, the coefficient of the quadratic term passes through zero. For each $x$, the symbol curve is the parabola $\operatorname{Re} z=$ $-\sin (x)(\operatorname{Im} z)^{2}$ described in the direction of decreasing imaginary part. Completing this curve in the usual manner by a semicircle at infinity, we see that its winding number is 1 about points to its right and 0 about points to its left. For any $\lambda$ in the domain bounded by the two parabolas $\operatorname{Re} z= \pm(\operatorname{Im} z)^{2}$, the winding number accordingly decreases by 1 at some value of $x$ in the interval $(-\pi,-\pi / 2)$ or $(\pi / 2, \pi)$, leading to an exponentially good wave packet pseudoeigenfunction. Figure 4 confirms this geometry for the case $h=0.1$.

From a dynamical point of view, a distinctive feature of (6.4) is that although the pseudospectra fill unbounded expanses of the right half-plane, all the eigenvalues lie on the neutrally stable imaginary axis. It is clear that despite the harmless-looking spectrum, this operator cannot generate a semigroup - $\left\|e^{t A}\right\|$ would have to be $\infty$ for all $t>0$. Benilov et al. argue that the underlying fluid mechanical problem is susceptible to a phenomenon of "explosive instability."

For a higher-order example, consider the fourth-order differential operator

$$
A_{h} u=h^{4} u_{x x x x}-h \sin (x) u_{x}, \quad x \in(-\pi, \pi),
$$

with periodic boundary conditions and symbol $f(x, k)=k^{4}+i \sin (x) k$. For $x=$ $-\pi / 2$, the symbol curve is the quartic $k^{4}-i k$, enclosing each point $\lambda$ inside with winding number 3 (once by the quartic itself, twice more by the fourth power of a large semicircle at $\infty$ ). As $x$ increases, for any $\lambda$ in this region, the winding number decreases to 2 when the curve crosses once and then to 1 as it crosses a second time. 

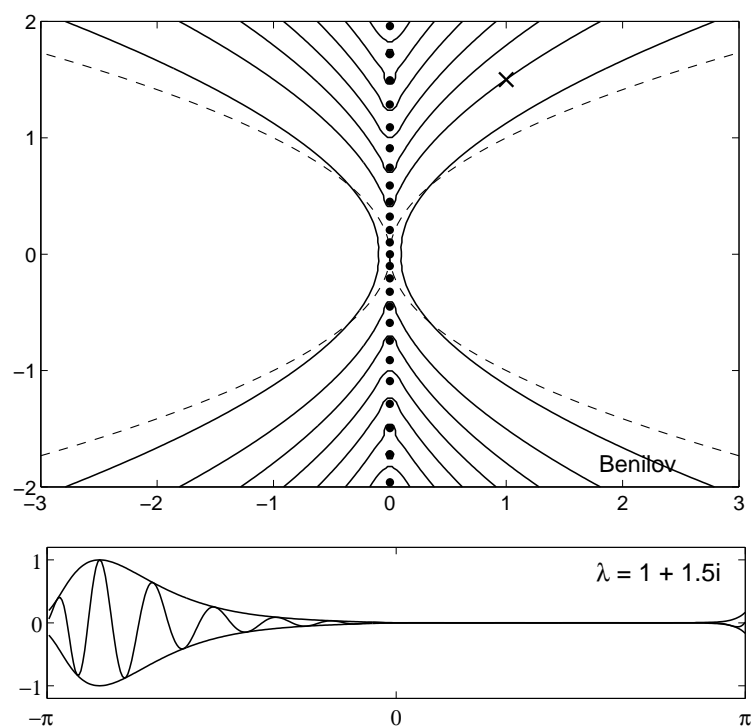

Figure 4. Above, eigenvalues and $\varepsilon$-pseudospectra of the operator (6.4) of Benilov, et al. [5] with $h=0.1$ and $\varepsilon=10^{-1}, \ldots, 10^{-7}$. By Theorem $1,\left\|\left(\lambda-A_{h}\right)^{-1}\right\|$ grows exponentially as $h \rightarrow 0$ for all $\lambda$ in the region between the two dashed parabolas. Below, an optimal pseudoeigenfunction for $\lambda=1+1.5 i$ (marked by a cross) with central position and wave number $x_{*}=\sin ^{-1}(4 / 9)-\pi \approx-2.68$ and $k_{*}=-1.5$.

Thus for each such $\lambda$ we expect exponentially good pseudomodes consisting of a pair of wave packets (Figure 5). In the special case $\operatorname{Im} \lambda=0$, both crossings occur at the same value of $x_{*}$. (Theorem 1 as written does not apply in this case, but that is an accident of wording, for in fact its proof is valid in such cases of multiple crossings so long as there is a net decrease in winding number.) In this special case there will be pseudomodes consisting of two wave packets superimposed at the same $x_{*}$ and with opposite values of $k_{*}$. This explains the lack of a smooth envelope in the figure.

We now move to a different type of example, in which the symbol curve at a particular value $x_{*}$ passes through the value $\lambda$ of interest for two different wave numbers $k_{*}$. In such cases Theorem 1 does not apply, but since our coefficients depend analytically on $x$, we can appeal instead to Theorem 1.2 of Dencker, Sjöstrand and Zworski [21], which ensures as before the existence of pseudoeigenfunctions, now localized in $k$ as well as $x$, that are exponentially good in the sense of (3.6).

Our fourth example is Davies's non-selfadjoint harmonic oscillator from $[\mathbf{1 5}, \mathbf{1 6}]$. Written with a small parameter $h$, we have

$$
A_{h} u=-h^{2} u_{x x}+i x^{2} u, \quad x \in(-\infty, \infty),
$$

with symbol $f(x, k)=k^{2}+i x^{2}$. For any fixed $x_{*} \in \mathbf{R}$, the symbol curve is the halfline $i x_{*}^{2}+[0, \infty)$ in the complex plane traversed from $\infty$ to $i x_{*}^{2}$ and back again to $\infty$. For each $\lambda$ along this half-line and corresponding choice of $x_{*}$, there are two values of $k_{*}$, one of which satisfies the twist condition (the one whose sign is the same as that of $\left.x_{*}\right)$. We can see this either by calculating the twist ratio $(\partial f / \partial x) /(\partial f / \partial k)=$ $i x / k$, or by thinking of sections of the symbol curve. We conclude that every $\lambda \in \mathbf{C}$ 


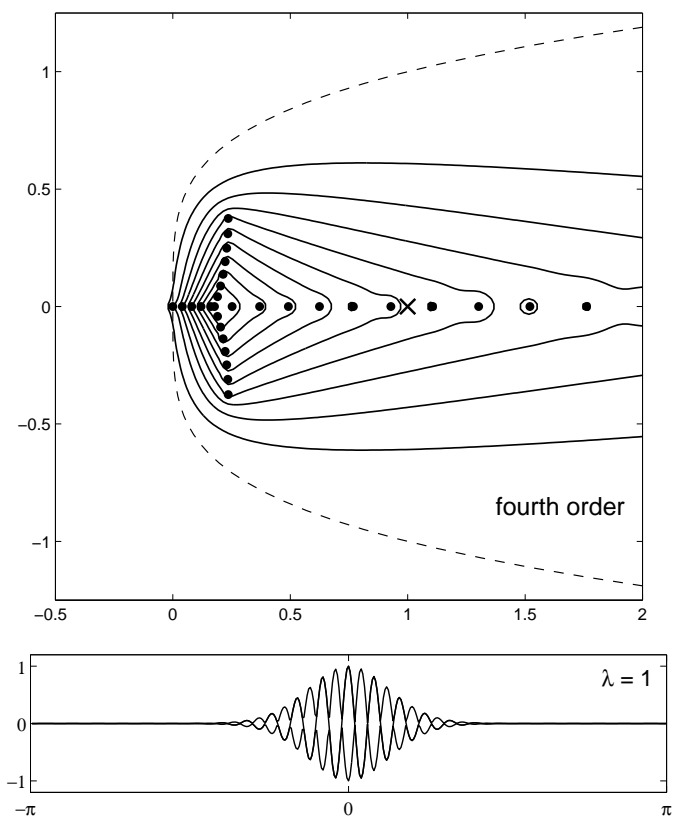

FIGURE 5. Above, eigenvalues and $\varepsilon$-pseudospectra of the fourth-order operator (6.5) with $h=0.04, \varepsilon=10^{-2}, \ldots, 10^{-10}$. By Theorem $1,\left\|\left(\lambda-A_{h}\right)^{-1}\right\|$ grows exponentially as $h \rightarrow 0$ for any $\lambda$ lying in the quartic region marked by the dashed line. Below, an optimal pseudomode for $\lambda=1$ (cross), with $x_{*}=0$ and $k_{*}$ taking both values 1 and -1 .

with $\operatorname{Re} \lambda>0, \operatorname{Im} \lambda>0$ is an $\varepsilon$-pseudoeigenvalue of $A_{h}$ for an exponentially small value of $\varepsilon$, as shown in Fig 6 . There are two values of $x_{*}$ for each $\lambda$, which explains why the optimal pseudomode in the figure consists of two wave packets rather than one.

It is worth commenting further on the significance of a case like Figure 6 in which two wave packets appear in a computed pseudomode. The arguments in this paper and in $[\mathbf{2 1}]$ construct exponentially good pseudomodes in the form of single wave packets, not double ones. The present case is special because there are two values of $x_{*}$ that are equally good for this construction: in some sense the multiplicity of the pseudoeigenvalue is two rather than the usual value of one. Thus it would be equally valid to show a psuedomode with just one wave packet on the left, or on the right; except that the optimal pseudomode, less than $0.003 \%$ better than these, is the odd function with two bumps. (Orthogonal to this would be another function with two bumps, but even instead of odd, and little different to the eye.)

Davies and Kuijlaars have analyzed the operator (6.6) in detail in [19] (with $i$ replaced by an arbitrary complex constant), basing their arguments on the theory of polynomials orthogonal with respect to a complex weight function. Among other results their Theorem 3 implies that the condition numbers $\kappa\left(\lambda_{n}\right)$ of the eigenvalues of (6.6) grow exponentially at the rate

$$
\lim _{n \rightarrow \infty} \kappa\left(\lambda_{n}\right)^{1 / n}=1+\sqrt{2},
$$

with eigenvalues indexed with increasing distance from the origin. Such a precise 

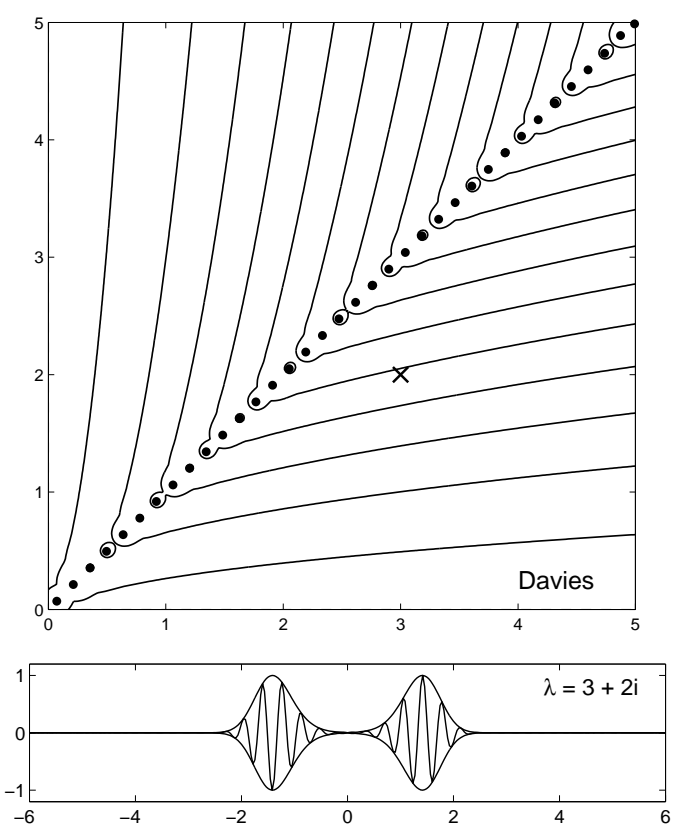

Figure 6. Above, eigenvalues and $\varepsilon$-pseudospectra of the Davies example (6.6) with $h=1 / 10, \varepsilon=10^{-1}, \ldots, 10^{-13}$. By the results of Dencker et al. $[\mathbf{2 1}],\left\|\left(\lambda-A_{h}\right)^{-1}\right\|$ grows exponentially as $h \rightarrow 0$ for any $\lambda$ lying in the first quadrant of the complex plane. Below, an optimal pseudoeigenfunction for $\lambda=3+2 i$ (cross), with $x_{*}= \pm \sqrt{2}$ and $k_{*}= \pm \sqrt{3}$.

estimate goes beyond the results presented here or in $[\mathbf{2 1}]$, where the constants are not specified.

Our next example is closely related to Davies's, the only difference being that the coefficient $i x^{2}$ is replaced by $i x^{3}$. This 'complex cubic oscillator' is a representative of a class of operators that have been discussed by Bender and others, starting from unpublished work of D. Bessis in 1995, for applications in non-Hermitian quantum mechanics $[3,4,20,25,31]$. The equation is

$$
A_{h} u=-h^{2} u_{x x}+i x^{3} u, \quad x \in(-\infty, \infty),
$$

with symbol $f(x, k)=k^{2}+i x^{3}$. Mathematically, this is much the same as the Davies example, but the pseudospectra fill the right half-plane instead of the first quadrant since $x^{3}$ ranges over all of $\mathbf{R}$ rather than just $[0, \infty)$ (Figure 7 ). Most of this literature is concerned with establishing properties of the eigenvalues of (6.7) and related operators and does not question their physical significance. Again, the results of Dencker, et al. apply to this operator, but Theorem 1 does not.

Another operator with the same flavour as those of Davies and Bender is

$$
A_{h} u=h^{2} u_{x x}+\alpha x^{2}-\gamma x^{4}, \quad x \in(-\infty, \infty),
$$

where $\alpha$ and $\gamma$ are parameters. This has no particular physical significnce, but the pseudospectra, for $\alpha=3+3 i$ and $\gamma=1 / 16$, are explored in [41]. As with the Cossu-Chomaz operator, there are significant transient effects, with all eigenvalues in the left half-plane but large resolvent norms in the right half-plane.

None of the examples we have presented were the first examples of variable coef- 

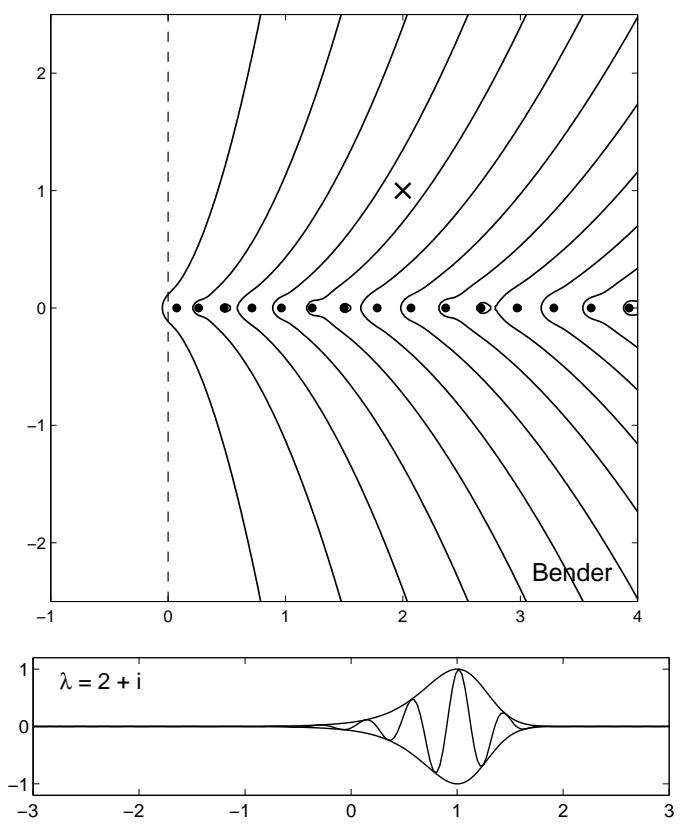

Figure 7. Above, eigenvalues and $\varepsilon$-pseudospectra of Bender's complex cubic oscillator (6.7) of $[\mathbf{3}, \mathbf{4}]$ with $h=0.1, \varepsilon=10^{-1}, \ldots, 10^{-13}$. By the results of Dencker et al., $\left\|\left(\lambda-A_{h}\right)^{-1}\right\|$ grows exponentially as $h \rightarrow 0$ for any $\lambda$ lying in the right half-plane. Below, an optimal pseudoeigenfunction for $\lambda=2+i$ (cross), with $x_{*}=1$ and $k_{*}=\sqrt{2}$.

ficient differential operators whose pseudospectra were computed numerically. That distinction belongs to the Orr-Sommerfeld and Airy operators, whose pseudospectra were computed together by Reddy, Schmid and Henningson in 1993 [34]. The Orr-Sommerfeld operator is in generalized eigenvalue form and does not quite fit the framework of this paper. The Airy operator is

$$
A_{h} u=h^{2} u_{x x}+i x u, \quad x \in[-1,1],
$$

with boundary conditions $u(-1)=u(1)=0$ and symbol $f(x, k)=-k^{2}+i x$. Figure 8 presents results corresponding to $h=0.02$. This operator has also been investigated by Stoller, Happer, and Dyson [39], Shkalikov [37], and Redparth [35].

\section{Robustness and structural stability}

For all of our six examples, we have a theorem to explain why exponentially good wave packet pseudoeigenfunctions had to appear: Theorem 1.2 of Dencker, Sjöstrand and Zworski [21] for all six, and Theorem 1 of this paper for just the first three, since the others had double-crossing symbol curves. Now the theorem of Dencker et al. requires smoothness of the symbol, whereas Theorem 1 does not. Therefore one might expect that the first three examples should be robust in the sense that the exponentially good pseudomodes persist if the operator coefficients undergo a nonsmooth perturbation, whereas the other three should be fragile.

We found confirming this prediction numerically to be challenging, for the computations underlying Figures 1-5 are based on spectral methods [42], a technology 

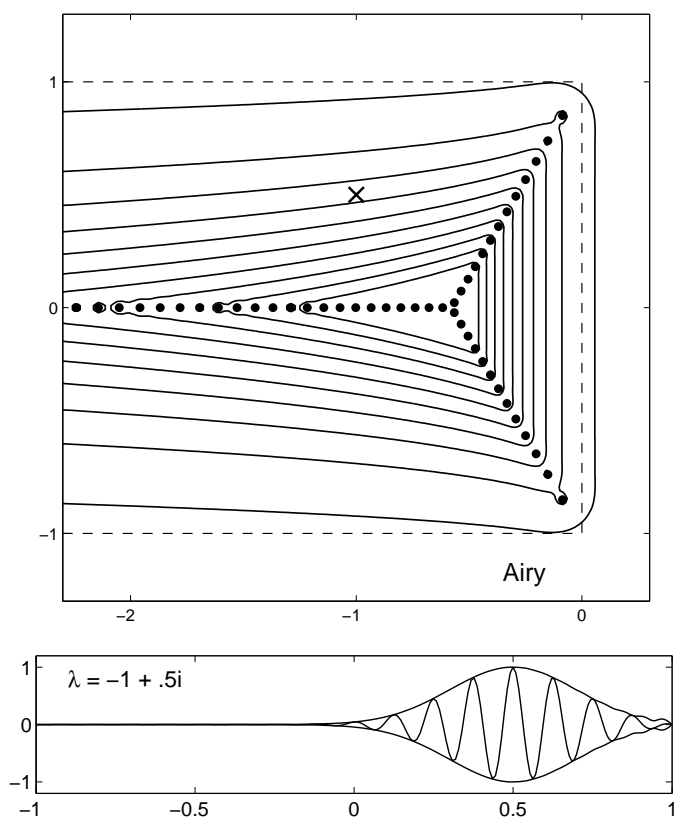

Figure 8. Above, eigenvalues and $\varepsilon$-pseudospectra of the Airy operator (6.9) with $h=0.02, \varepsilon=10^{-1}, \ldots, 10^{-13}$. By the results of Dencker, et al., $\left\|\left(\lambda-A_{h}\right)^{-1}\right\|$ grows exponentially as $h \rightarrow 0$ for any $\lambda$ lying in the dashed half-strip. Below, an optimal pseudoeigenfunction for $\lambda=-1+0.5 i$ (cross), with $x_{*}=0.5$ and $k_{*}=-1$.

that relies on smooth functions for its power, whereas if one reverts to simpler finite differences or finite elements, based on weaker smoothness assumptions, the accuracy is usually too low to resolve $\varepsilon$-pseudospectra for small values of $\varepsilon .^{\dagger}$ The compromise we eventually reached was to continue to use spectral methods but to choose perturbations that are somewhat smooth. We perturbed each ODE coefficient by multiplying it by a function having five but not six continuous derivatives, with amplitude varying between 0.9 and 1.1. The results appear in Figure 9. As predicted, three of the cases shown are robust and three are fragile, with pseudospectra distorted almost beyond recognition by this $C^{5}$ perturbation. Theorem 1 ensures that the robust cases would in fact stand up to far rougher perturbations; it is just difficult to verify such cases numerically.

The same distinction between robust and fragile pseudospectra arises for twisted Toeplitz matrices and is discussed in [43]; see Figs. 7.4 and 8.1 of that paper.

\footnotetext{
${ }^{\dagger}$ Indeed, an interesting subtlety arises here. Suppose one discretizes a smooth differential equation by a finite difference approximation on a uniform grid. The result is a twisted Toeplitz matrix. However, if the symbol curve for the differential equation has no crossings $f\left(x_{*}, k\right)=f\left(x_{*}, k_{*}\right)$ for $k \neq k_{*}$, so that Theorem 1 is applicable, this does not imply the same property for the matrix approximation. Thus even when a differential equation has pseudospectra that are robust with respect to perturbations, those of its finite difference approximations will typically be fragile.
} 

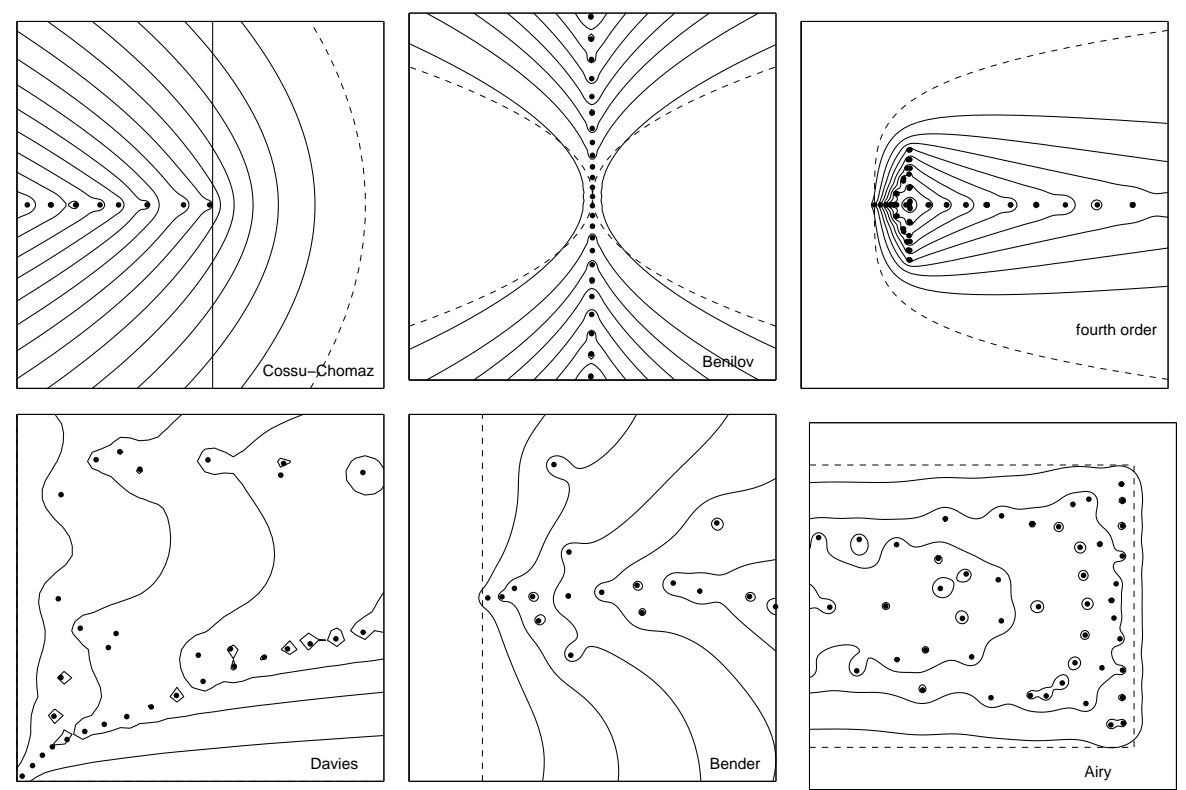

FIGURE 9. A repetition of our six examples with each operator coefficient modified by a $C^{5}$ multiplicative perturbation. The first three have simply-crossing symbol curves, and the perturbation has little effect on the pseudospectra (Theorem 1). For the next three, it changes them completely.

\section{Generalizations}

The results we have presented are restricted to ordinary differential operators, in one space dimension. If the symbol is smooth, the WKBJ/microlocal approach of Dencker, et al. [21] generalizes to partial differential or pseudodifferential operators, and their results are presented in this framework. On the other hand it is not clear whether our results for nonsmooth symbols can be generalized in this way.

Another kind of generalization would concern discontinuities of coefficients or boundaries of the domain. For twisted Toeplitz matrices, such generalizations are considered at length in $[\mathbf{4 3}]$, where it is pointed out that earlier results of Reichel and Trefethen [36] on pseudomodes pinned at boundaries can be derived as a special case of the wave packet theory for discontinuous coefficients. For differential operators, one could presumably work out analogous results, deriving the results of Reddy [33] and Davies [17] for boundary pseudomodes from a wave packet theory. Such an approach would make it clear that the existence of boundary pseudomodes does not depend upon constant coefficients, a matter first taken up in [18].

Related to boundaries and discontinuities is the matter of the antitwist condition, which we mentioned in Theorem 1 but not illustrated in our examples. Suppose one has a family of operators such that as $x$ increases, the symbol curves sweep across $\lambda \in \mathbf{C}$ to decrease the winding number but do not sweep back again to increase the winding number. Then $\lambda$ will be an exponentially good pseudoeigenvalue with wave packet pseudoeigenfunctions, whereas for the transposed operators (complex 
conjugate of the adjoint), the pseudoeigenfunctions will be localized at the boundary.

Acknowledgements. Much of this article was completed during a visit to the National University of Singapore in December 2003; I thank Toh Kim-Chuan and and Lee Seng-Luan and the other stimulating mathematicians at the NUS for supporting this visit. Concerning the mathematics of wave packet pseudomodes I have benefited from interactions with many people, of whom three have been especially important: Jon Chapman of Oxford University, Brian Davies of King's College London and Maciej Zworski of the University of California, Berkeley.

\section{References}

1. A. Aslanyan and E. B. Davies, 'Spectral instability for some Schrödinger operators', Numer. Math. 85 (2000) 525-552.

2. U. M. Ascher, R. M. M. Mattheij and R. D. Russell, Numerical Solution of Boundary Value Problems for Ordinary Differential Equations (SIAM, Philadelphia, 1995).

3. C. M. Bender and S. Boettcher, 'Real spectra in non-Hermitian Hamiltonians having $\mathcal{P} \mathcal{T}$ symmetry', Phys. Rev. Lett. 80 (1998) 5243-5246.

4. C. M. Bender, S. Boettcher and P. N. Meisinger, 'P $\mathcal{T}$-symmetric quantum mechanics', J. Math. Phys. 40 (1999) 2201-2229.

5. E. S. Benilov, S. B. G. O'Brien and I. A. Sazonov, 'A new type of instability: explosive disturbances in a liquid film inside a rotating horizontal cylinder', J. Fluid Mech. 497 (2003), 201-224.

6. D. Borthwick and A. URIBE, 'On the pseudospectra of Berezin-Toeplitz operators', to appear.

7. L. S. Boulton, 'Non-self-adjoint harmonic oscillator, compact semigroups and pseudospectra', J. Oper. Th. 47 (2002) 413-429.

8. S. J. Chapman, 'Subcritical transition in channel flows', J. Fluid Mech. 451 (2002) 35-97.

9. C. Chicone and Y. Latushkin, Evolution Semigroups in Dynamical Systems and Differential Equations, Amer. Math. Soc., Providence, RI 1999.

10. J. M. Chomaz, P. Huerre and L. G. Redekope, in Proceedings of the Sixth Symposium on Turbulent Shear Flows, Toulouse, France, 1987, pp. 3.2.1-3.2.6, 1987.

11. E. A. Coddington and N. Levinson, Theory of Ordinary Differential Equations (McGrawHill, New York, 1955).

12. W. A. Coppel, Dichotomies in Stability Theory, Lect. Notes in Math., v. 629, SpringerVerlag, Berlin, 1978.

13. C. Cossu and J. M. Chomaz, 'Global measures of local convective instabilities', Phys. Rev. Lett. 78 (1997), 4387-4390.

14. Ju. L. DALECKII and M. G. KRein, Stability of Solutions of Differential Equations in Banach Space, Amer. Math. Soc., Providence, RI, 1974.

15. E. B. Davies, 'Pseudo-spectra, the harmonic oscillator and complex resonances', Proc. Roy. Soc. Lond. A 455 (1999) 585-599.

16. E. B. Davies, 'Semi-classical states for non-self-adjoint Schrödinger operators', Comm. Math. Phys. 200 (1999) 35-41.

17. E. B. DAvies, 'Pseudospectra of differential operators', J. Oper. Th. 43 (2000), 243-262.

18. E. B. DAVIES, 'Semiclassical analysis and pseudospectra', manuscript, 2004.

19. E. B. DAvies and A. B. J. KuiJlaARs, 'Spectral asymptotics of the non-self-adjoint harmonic oscillator', J. Lond. Math. Soc., to appear.

20. E. Delabaere and D. T. Trinh, 'Spectral analysis of the complex cubic oscillator', J. Phys. A 33 (2000) 8771-8796.

21. N. Dencker, J. Sjöstrand and M. Zworski, 'Pseudospectra of semiclassical (pseudo-) differential operators', Comm. Pure Appl. Math. 57 (2004) 384-415.

22. G. Domokos and P. Holmes, 'On nonlinear boundary-value problems: ghosts, parasites and discretizations', Proc. Roy. Soc. Lond. A 459 (2003) 1535-1561.

23. M. Dimassi and J. SuÖstrand, Spectral Asymptotics in the Semi-Classical Limit, Lond. Math. Soc. Lect. Notes, v. 268, Cambridge U. Press, Cambridge, 1999.

24. M. Embree and L. N. Trefethen, Pseudospectra Gateway, web site at http://www . comlab. ox.ac.uk/pseudospectra.

25. C. R. HANDY, 'Generating converging bounds to the (complex) discrete states of the $P^{2}+$ $i X^{3}+i \alpha X$ Hamiltonian', J. Phys. A 34 (2001) 5065-5081. 
26. L. Hörmander, 'Differential equations without solutions', Math. Ann. 140 (1960) 169-173.

27. H. J. LANDAU 'On Szegö's eigenvalue distribution theory and non-Hermitian kernels', J. d'Anal. Math. 28 (1975), 335-357.

28. H. LEWY, 'An example of a smooth linear partial differential equation without solution', Ann. Math. 66 (1957) 155-158.

29. A. Martinez, An Introduction to Semiclassical and Microlocal Analysis (Springer-Verlag, New York, 2002).

30. J. L. MAsserA and J. J. SchäFer, Linear Differential Equations and Function Spaces, Academic Press, New York, 1966.

31. G. A. Mezincescu, 'Some properties of eigenvalues and eigenfunctions of the cubic oscillator with imaginary coupling constant', J. Phys. A 33 (2000) 4911-4916.

32. K. Pravda-Starov, 'A general result about the pseudo-spectrum of Schrödinger operators', Proc. Roy. Soc. Lond. A 460 (2004) 471-477.

33. S. C. REDDY, Pseudospectra of Wiener-Hopf integral operators and constant-coefficient differential operators, J. Int. Eqs. Applics. 5 (1993), 369-403.

34. S. C. Reddy, P. J. Schmid and Dan S. Henningson, 'Pseudospectra of the Orr-Sommerfeld operator', SIAM J. Appl. Math. 53 (1993) 15-47.

35. P. REDPARTH, 'Spectral propoerties of non-self-adjoint operators in the semi-classical regime, J. Diff. Eqs. 177 (2001) 307-330.

36. L. ReicheL and L. N. TREFEthen 'Eigenvalues and pseudo-eigenvalues of Toeplitz matrices,' Lin. Alg. Applics. 162/164 (1992), 153-185.

37. A. Shkalikov, 'The limit behavior of the spectrum for large parameter values in a model problem', Math. Notes 62 (1997) ??-??

38. M. Shub, Global Stability of Dynamical Systems, Springer-Verlag, New York, 1987.

39. S. D. Stoller, W. Happer and F. J. Dyson, 'Transverse spin relaxation in inhomogeneous magnetic fields', Phys. Rev. A 44 (1991) 7459-7477.

40. L. N. Trefethen, 'Pseudospectra of linear operators', SIAM Review 39 (1997), 383-406.

41. L. N. Trefethen, 'Computation of pseudospectra', Acta Numerica 8 (1999) 247-295.

42. L. N. Trefethen, Spectral Methods in MATLAB (SIAM, Philadelphia, 2000).

43. L. N. Trefethen and S. J. Chapman, 'Wave packet pseudomodes of twisted Toeplitz matrices', Comm. Pure Appl. Math., to appear in 2004.

44. L. N. Trefethen, A. E. Trefethen, S. C. Reddy and T. A. Driscoll, 'Hydrodynamic stability without eigenvalues', Science 261 (1993), 578-585.

45. S. Wiggins, Introduction to Applied Nonlinear Dynamical Systems and Chaos, SpringerVerlag, Berlin, 1990.

46. T. G. WRIGHT, EigTool software package, http://www.comlab.ox.ac.uk/pseudospectra/ eigtool/.

47. M. Zworski, 'A remark on a paper of E. B. Davies', Proc. Amer. Math. Soc. 129 (2001) $2955-2957$

48. M. ZwORSKI, 'Numerical linear algebra and solvability of partial differential equations', Comm. Math. Phys. 229 (2002), 293-307.

Lloyd N. Trefethen

Oxford University Computing

Laboratory

Wolfson Bldg., Parks Road

Oxford OX1 3QD

LNT@comlab.ox.ac.uk 